\title{
PERCEPÇÃO DE RISCO DE ADOLESCENTES ESCOLARES NA RELAÇÃO CONSUMO DE ÁLCOOL E COMPORTAMENTO SEXUAL
}

\author{
Francisco Jucier Luz SAM PAIO FIL H O ${ }^{a}$, Pedro Ricardo M esquita deSOUSA ${ }^{\mathrm{b}}$, N eiva F rancenely Cunha VIEIRAc,
} $M$ aria de F átima Bastos N ÓBRE GA d, Fabiane do A maral GU BE RT e, Patrícia N eyva da Costa PIN H E IRO ${ }^{f}$

\section{RESUM 0}

0 uso de álcool tem sido uma das principais causas desencadeadoras de situações de vulnerabilidade na adolescência. 0 estudo tem como objetivo analisar a percepção de risco de adolescentes acerca da relação consumo de álcool/comportamento sexual. Os sujeitos foram dez adolescentes entre 14 e 19 anos, estudantes de escola pública em Fortaleza, Ceará. Estudo exploratório-descritivo, de abordagem qualitativa, fundamentado na técnica de Grupo focal, sistematizado por meio de cinco oficinas. Os adolescentes reconhecem o risco entre o consumo de ál cool e comportamento sexual, e destacam que 0 ato de beber facilita as relações entre os pares e apontam a influência da mídia neste processo. Conhecer a percepção dos adolescentes e sua relação com o abuso de álcool e suas conseqüências é relevante para a implementação de políticas de prevenção e combate ao consumo de bebidas al coólicas nesta etapa de crescimento e desenvolvimento.

D escritores: A dolescente. Consumo de bebidas al coólicas. Comportamento sexual.

\section{RESUMEN}

E I consumo de alcohol ha sido una las principales causas desencadenantes de situaciones de vulnerabilidad en la adolescencia. E I estudio tiene como objetivo anal izar la percepción de riesgo sobrela relación del consumo dealcohol de los adolescentes y el comportamiento sexual. L os sujetos fueron diez adolescentes con edades entre 14 y 19 años, estudiantes de una escuela pública en Fortaleza, C eará, B rasil. E studio ex ploratorio y descriptivo, basado en la técnica de grupos focales y sistematizado a través de cinco talleres. $L$ os adolescentes reconocen el riesgo entreel consumo de alcohol y el comportamiento sexual y hacen hincapié en que la bebida facilita las relaciones entre parejas sexuales e indican la influencia de los medios de comunicación en este proceso. L a percepción de los adolescentes y su relación con el abuso del alcohol es particularmenterelevante para la aplicación de las políticas para prevenir el consumo de al cohol en esta etapa de crecimiento y desar rollo.

D escriptores: A dolescente Consumo de bebidas alcohólicas. Conducta sexual.

T ítulo: P ercepción de riesgo de adolescentes en escuela relacionados con el al cohol y el comportamiento sexual.

\section{ABST RACT}

A lcohol use has been a major precipitating cause of situations of vulnerability in adolescence The study aims to analyze the risk perception about the relationship of adol escent al cohol use and sexual behavior. The subjects were ten adolescents betw een 14 and 19, students from a public school in Fortaleza, Ceará, B razil. E xploratory and descriptive qualitative approach based on the technique of focus group and systematized by means of five workshops. T eenagers recogniz e the risk betw een alcohol consumption and sexual behavior and emphasize that drinking facilitates the relations between sexual peers and indicate the influence of media in this process. The perception of adolescents and their relation to alcohol abuse and its consequences is particularly rel evant to the implementation of public policies for preventing and fighting alcohol consumption at this stage of growth and development.

Descriptors: A dolescent. Alcohol drinking. Sexual behavior.

T itle: Perception of risk of school adolescents in relation to alcohol consumption and sexual behavior.

\footnotetext{
a Enfermeiro graduado pela U niversidade Federal do Ceará (U FC), Fortaleza, Ceará, Brasil.

b Enfermeiro, Bolsista da Escola de Saúde Pública do Ceará, Fortaleza, Ceará, Brasil.

c PhD em E ducação em Saúde, D ocente do Programa de Pós-G raduação em Enfermagem da U FC, Bolsista de Produtividade em Pesquisa do Conselho N acional de D esenvolvimento Científico e Tecnológico (CN PQ) - nível 2, Fortaleza, Ceará, Brasil.

d Enfermeira, Doutoranda em Enfermagem da UFC, Serviço de Educação Continuada em Enfermagem, H ospital U niversitário Walter Cantídio, Fortaleza, Ceará, Brasil.

e Enfermeira, D outoranda em Enfermagem da UFC, Bolsista da Coordenação de A perfeiçoamento de Pessoal de Nível Superior (CAPES), Fortaleza, Ceará, Brasil.

f D outora em Enfermagem, D ocente do Curso de Graduação em Enfermagem da U FC, Fortaleza, Ceará, Brasil.
} 


\section{INT RODUÇÃO}

A dolescência é uma etapa crucial do desenvolvimento do indivíduo, e marca não apenas a aquisição da imagem corporal definitiva como também a estruturação final da personalidade. A Organização M undial da Saúde (OM S) considera esta fase como a segunda década da vida, de 10 a 19 anos. Já a lei brasileira considera adolescência, a faixa etária entre 12 e 18 anos; assim, há uma divergência entre a fixação etária do Estatuto da Criança e do A dolescente (ECA) e da OM S, também adotada pelo M inistério da Saúde ${ }^{(1)}$.

A tualmente, 1,7 bilhões de pessoas (mais do que a quarta parte dos habitantes do planeta) são adolescentes e jovens, sendo que $86,0 \%$ dos indivíduos deste grupo etário habitam em países em desenvolvimento ${ }^{(2)}$. $\mathrm{N}$ o Brasil a Pesquisa $\mathrm{N}$ acional por A mostra de Domicílios (PNAD) do ano de 2008 evidencia que quase metade $(49,6 \%)$ das famílias brasileiras, conta com, pelo menos, um de seus componentes com idade até 14 anos. D estaca também, crescimento na taxa de adolescentes que freqüentam a escola na última década, atingindo, em 2007, $81,7 \%{ }^{(3)}$.

A população escolar destacando adolescentes entre 11 e 19 anos, adoece menos que outros grupos etários e, de fato, tem taxas de morbidade e mortalidade mais baixas que da população em geral. M as um olhar mais aprofundado aponta para um aumento no número de adolescentes que adoecem pelo abuso de álcool, que associados a fatores socioeconômicos e culturais, têm profundas repercussões na qualidade de vida e saúde ${ }^{(4)}$.

0 uso e 0 abuso de álcool tem sido uma das principais causas desencadeadoras de situações de vulnerabilidade na adolescência e juventude, a exemplo dos acidentes, suicídios, violência, gravidez não planejada e a transmissão de doenças por via sexual (5). D eacordo com o V L evantamento N acional com Estudantes realizado em 2004 pelo Centro Brasileiro de Informações sobre D rogas Psicotrópicas (CE BRID), 65,2\% dos estudantes relataram uso de álcool; 44,3\% nos últimos 30 dias; $11,7 \%$ uso freqüente, ou seja, seis ou mais vezes no mês; e 6,7\% uso pesado, isto é, 20 ou mais vezes no último mês ${ }^{(6)}$.

No que se refere ao comportamento sexual de risco o uso de álcool é considerado um dos principais fatores que incidem negativamente neste contexto influenciando inclusive, o início precoce de atividade sexual, não uso de preservativo, pagamento por sexo e, inclusive, prostituição ${ }^{(7)}$. Portanto, por se tratar de uma droga de fácil acesso e lícita, o álcool se apresenta como um dos principais antagonistas da vivência saudável da sexualidade, o que contribui para um aumento da vulnerabilidade em relação às doenças sexualmente transmissíveis (DST ). D esse modo, nota-se que a compreensão dos problemas relacionados ao consumo de álcool entre adolescentes deve se estender para além da prevalência do uso, e considerar também os diversos fatores que influenciam este comportamento.

Conhecer a percepção dos adolescentes acerca dos sentimentos presentes nesta fase e sua relação com o consumo de ál cool e suas conseqüências é particularmente relevante para a implementação de políticas públicas de prevenção e combate ao consumo de bebidas alcoólicas, especial mente porque este grupo encontra-se em pleno crescimento e desenvolvimento físico e mental e os danos causados pelo álcool podem ser irreparáveis. Partindo dessas premissas, o presente estudo tem como objetivos analisar a percepção de adol escentes acerca da vulner abilidade e risco na relação consumo de álcool e comportamento sexual.

\section{PERCURSO METODOLÓGICO}

Trata-se de um estudo exploratório e descritivo, com abordagem qualitativa, realizado em uma escola pública de Fortaleza, Ceará. A abordagem qualitativa se justifica por ser a forma mais adequada para responder aos objetivos propostos, na medida em que procura centrar a atenção na especificidade, no individual, almejando sempre a compreensão dos fenômenos estudados ${ }^{(8)}$.

Os sujeitos do estudo foram dez adolescentes na faixa etária entre 14 e 19 anos. Os critérios de seleção dos participantes foram: estar regularmente matriculados na instituição, ter disponibilidade para participar das atividades, interesse pela temática, assiduidade nas aulas e autorização dos pais.

A coleta de informações ocorreu entre os meses de março e maio de 2009. A técnica de grupo focal foi utilizada a fim de desvelar as opiniões e percepções dos adolescentes, por meio de cinco oficinas com duração de uma hora e trinta minutos. 0 grupo focal pode ser entendido como uma entrevista de grupo, embora não no sentido de ser 
um processo onde se alternam perguntas do pesquisador e resposta dos participantes. Difer entemente, a essência dessa técnica reside justamente na relação de interação entre seus participantes, no processo de coleta de dados. A coleta de informações se desenvolve a partir de tópicos que são for necidos pelo pesquisador ou moderador do grupo ${ }^{(9)}$.

Para conduzir o grupo focal há um moderador, o qual assegura por meio de uma intervenção discreta e firme, que o grupo cubra os tópicos de interesse do estudo da maneira menos diretiva possível ${ }^{(9)}$. No estudo os moderadores foram dois pesquisadores treinados para a coleta de informações com proficiência na temática, os quais observaram a conduta do grupo, auxiliando na anotação dos acontecimentos-chave.

Com vistas a aprofundar o processo de coleta de informações e interação entre pesquisadores e adolescentes utilizou-se consoante a técnica de grupo focal a metodologia de oficinas em dinâmica de grupo, prática de intervenção psicossocial adaptável a diver sos contextos. A oficina tem suas bases e forma de organização originárias da pesquisa-ação, grupos operativos e pedagogia da autonomia ${ }^{(10)}$. D esta forma, as oficinas educativas tiveram as seguintes etapas:

a) levantamento - necessidades dos adolescentes, tendo em vista as prioridades, detectadas no momento de apresentação dos objetivos do estudo;

b) planejamento - ciclo de cinco oficinas, nas quais utilizamos técnicas de sensibilização, dinamização, comunicação e reflexão, a fim de propiciar a formação de vínculo grupal e estimular o debate nos grupos focais;

c) execução - implementação das oficinas e grupo focal, divididas em temas centrais: características do adolescer; DST e Vírus da Imunodeficiência Adquirida (HIV); efeitos do álcool sobre o comportamento sexual; mídia e sua influência na sexualidade do adolescente. Esta etapa baseouse em exposição dialogada e uso de materiais educativos preconizados pelo M inistério da Saúde;

d) avaliação - encontro final com adolescentes a fim de validar os achados do estudo, resgatando todas as oficinas realizadas e depoimentos registrados.

As informações submeteram-se à técnica de categorização dos discursos, que é um processo do tipo estruturalista que comporta duas etapas: 0 inventário, que é 0 ato de isolar os elementos, e a classificação, que é a divisão de forma organizada dos elementos da mensagem ${ }^{(8)}$. Em síntese podemos ordenar as idéias e os fatos segundo as semel hanças. E mergiram do estudo três categorias: Características da adolescência; Ingestão de Álcool e Comportamentos de Risco Sexual e A mídia como fator de risco para o consumo de álcool.

0 estudo respeitou os aspectos legais e éticos que envolvem pesquisas com seres humanos, conforme a Resolução 196/ 96 do Conselho N acional de Saúde ${ }^{(11)}$. A pesquisa foi aprovada pelo Comitê de Ética em Pesquisa da U niversidade Federal do Ceará (COM EPE), sob protocolo $n^{\circ} 118 / 08$.

\section{RESULTADOSE DISCUSSÕES}

\section{Características da adolescência}

O conteúdo presente nessa categoria referese à compreensão dos participantes sobre a adolescência, caracterizando-a como uma etapa de dificuldades e barreiras que seriam intrínsecas a essa fase do desenvolvimento.

É importante ressaltar que os adolescentes eram provenientes de famílias com renda familiar variando entre um e dois salários mínimos, seis adolescentes eram do sexo masculino e quatro do sexo feminino. Todos eram residentes próximos a escola selecionada, ou seja, um bairro da periferia da capital. Em relação à procedência dos jovens, três eram do interior do estado. A seguir, depoimento acerca do sentimento de vivenciar esta fase da vida:

\section{Ser adolescente é muito difícil (Cebolinha).}

Essa fala pode ser atribuída ao fato de 0 adolescente se perceber como alguém que está deixando a infância e está prestes a ingressar na vida adulta, sentindo-se, portanto, inseguro pela dificul dade de definir-se como criança ou adulto ${ }^{(12)}$. Outra característica oriunda dos discursos foi acerca do desejo de experimentar o novo.

A dolescente gosta de se divertir, de conhecer coisas novas (M agali).

Vivenciar situações novas e diferentes não é somente um grande desafio, mas pode ser 0 determinante da situação de adolescente. I sso porque essas situações abrem a possibilidade de testar os 
próprios limites e de experimentar "emoções inusitadas". A falta de maturidade do(a) adolescente, somada à curiosidade de experimentar o novo, consumir álcool, por exemplo, e à perspectiva do desafio resulta, quase que invariavel mente, em um dano, advindo daí uma situação de vulnerabilidade ${ }^{(13)}$.

0 aumento da vaidade é perceptível na adolescência, sendo o sexo feminino, o gênero que mantém uma relação mais íntima com essa característica, segundo os depoimentos:

[ ...] a genteéligada na moda, tenta andar arrumada, perfumada e tal. A gente começa a se preocupar com a aparência (Rosinha).

A vaidade se apresenta como um fator construtor da identidade do jovem, pois o corpo pode ser considerado a tela em que ele vai representar as suas subjetividades, valores criados em nossa sociedade, como a moda, e a inserção em determinado grupo social, além de ser uma forte manifestação da sexualidade no que diz respeito ao jogo de sedução entre os sexos. Por vaidade, os adolescentes muitas vezes consomem bebidas alcoólicas, o que faz com que se sintam diferentes, mais alegres, descontraídos e corajosos ${ }^{(13)}$.

A adolescência também é caracterizada por ser uma fase de conflitos, principalmente no campo do relacionamento interpessoal. Os conflitos presentes são ocasionados pelas grandes alterações de humores que sofrem nesta fase. A té certo ponto, eles se fazem necessários para a aquisição da identidade adulta.

[ ...] nós tivemos dificuldade de entrar em acordo com as meninas (Rolo).

$\mathrm{Na}$ adolescência os relacionamentos são caracterizados por mudanças, tendo picos de instabilidade, muitas vezes por não aceitar as normas e imposições dos pares. E sse comportamento é típico deste período, pois o adolescente deseja a sua almejada independência, procurando por novos valores e padrões de comportamento a partir do relacionamento e comunicação com novos grupos.

\section{Ingestão de álcool e comportamento sexual de risco}

N essa categoria, percebe-se que os participantes sentem-se expostos a riscos quando ingerem álcool:
$M$ uitas vez es quando a gente bebee pega a moto do pai escondida [ ...] pode acabar sofrendo um acidente. A gente sabe queéerrado, mas a vontadeé maior (Penadinho).

0 pensamento abstrato, ainda imaturo nos participantes, faz com que se sintam invulneráveis, se expondo a riscos sem prever suas consequências. Instáveis e susceptíveis a influências grupais, têm acrescido a isso o consumo de álcool como fator potencializador de comportamentos de risco devido ao sentimento de onipotência(14).

0 adolescente, no plano cognitivo, tem a dificuldade no acesso ao raciocínio formal, a dificuldade em fazer escolhas racionais em longo prazo e a dificuldade em refletir sobre todas as consequências dos seus atos, fazendo com que, muitas vezes, tenha uma percepção distorcida do risco real da infecção pelo HIV nas relações sexuais, pensando que este é um perigo impossível ou altamente improvável. E ssa percepção de risco, que já é distorcida normalmente na adolescência, é agravada com o consumo de bebidas al coólicas.

A fala dos adolescentes merece destaque ao apontar uma situação de risco relacionado ao consumo de álcool:

[ ...] pra não ficar defora, tem que beber mesmo às ve zes e até pra dar coragem pra chegar em uma menina, numa festa (Cebolinha).

Estudo realizado pelo Núcleo de Prevenção à Aids (NU PAIDS) observou que o consumo de bebidas alcoólicas estimula a atividade sexual, uma vez que logo após o uso do álcool a impressão dos adolescentes é de que a conquista do sexo oposto fica mais fácil, a libido é maior e o desempenho na relação sexual melhora ${ }^{(15)}$.

Para compreender o risco existente em relação ao uso de álcool e possível atividade sexual dos adolescentes, deve-se levar em conta também o ambiente no qual a bebida é utilizada, ou seja, em festas, bares, lugares de grande aglomeração de jovens, o que favorece 0 encontro de um possível parceiro sexual.

0 álcool e/ ou drogas ilusoriamente supre tudo o que desejam, dando a idéia de que são invulneráveis a quaisquer riscos e sofrimentos. Imunes ao perigo, eles não ponderam muito antes de se exporem a situações de perigo, nem se previnem em relação às DST: 
Se estiver muito bêbado, você nem lembra se está com camisinha ou não, vai de qualquer jeito, vai bem de sistir na hora lá (Penadinho).

[ ...] às vezes esquece de usar a camisinha, porque esta muito bêbado (Cascão).

A dolescentes que usam ál cool, são sexual mente mais ativos, têm maior número de parceiros e iniciam a atividade sexual precocemente ${ }^{(16)}$. 0 álcool causa um efeito desinibitório que facilita a relação sexual e, ao mesmo tempo, diminui a intenção de uso do preservativo, configurando-se assim, como um fator comprometedor da vivência saudável da sexualidade destes jovens.

É relevante destacar também que diante deste cenário a influência do ambiente familiar e social é fator condicionante para o uso de álcool, já que 0 adolescente começa a observar o comportamento dos pais desde muito cedo, e com isso percebe que situações de al egria e prazer estão relacionadas ao consumo de álcool. No decorrer de sua vida, repetirá esse comportamento, relacionandoo sempre às situações de descontração, desafio e coragem, nunca a um vício devastador ${ }^{(14)}$.

\section{A mídia como fator de risco para o consumo de álcool}

D urante a realização das oficinas, o tópico que discutiu a influência da mídia no início e fortalecimento da prática de consumo de álcool, foi um dos mais debatidos entre os alunos. Essa categoria mostra-se relevante no sentido de auxiliar o entendimento dos fatores que levam o adolescente a ingerir bebidas al coólicas.

Além da veiculação de propagandas de bebidas alcoólicas acontecerem em qualquer horário, há ainda a associação direta entre o consumo de bebidas e o sucesso, a sexualidade e participação de artistas nacional mente conhecidos e identificados com o público jovem. 0 seguinte depoimento revela esta realidade:

$\mathrm{N}$ a propaganda de cer veja, sempre tem mulher de biquíni, mulheres gatas, perto do cara quetá bebendo [ ...] e quando a gente bebe [...] a gente pensa nisso ( $\mathrm{Ce}$ bolinha).

É nítida a influência que a mídia exerce sobre a população e o indivíduo quando exposto às propagandas, tenderá a associar o consumo do ál- cool com prazer sempre que se colocar em uma situação ou ambiente que recorde as cenas vivenciadas nas propagandas ${ }^{(17)}$. 0 álcool passa a fazer parte da própria auto-imagem, a constituir um estilo, um jeito de ser, reforçando ainda mais positivamente a escolha pelo produto.

Características como quão atraentes as propagandas são para os jovens (principalmente as de cerveja) e sua exposição a elas estão relacionadas com uma maior expectativa de consumo futuro e possivel mente com um consumo maior e mais precoce por adolescentes.

$\mathrm{N}$ esse sentido, as imagens são criadas com 0 objetivo de "seduzir o sujeito" nas mais diversas faixas etárias. Os meios de comunicação em massa "retocam" a realidade de maneira a torná-la mais atraente, existe um culto à imagem. I sso é resultado do sistema capitalista dessa nova era, o qual transformou o consumo em uma instituição que determina um conjunto de valores com função de integ ral ização e controle social . 0 sentido de "ter" passa a substituir o sentido de "ser", para ser alguém socializado e inserido em um grupo social você precisa ter certas coisas, no caso dos adolescentes, para eles estarem inseridos em seu grupo eles precisam beber, ou ter a bebida alcoólica no seu cotidiano(18).

O M inistério da Saúde posiciona-se em relação às propagandas de bebidas alcoólicas no sentido de controlar sua veiculação, enfatiza a urgência de adoção de medidas visto que reconhece que o consumo de álcool entre jovens contribui decisivamente para situações de sexo desprotegido ${ }^{(19)}$.

$M$ as o ponto que deve ser mais enfatizado é relativo ao papel da família no combate a influência da mídia, a qual é referencial comportamental básico para o jovem, e ela pode influenciar o consumo tanto no plano da experimentação quanto no regular. A atitude mais ou menos crítica dos pais, bem como suas próprias pautas e parâmetros de consumo, podem atenuar ou reforçar a influência de instituições como agentes desencadeadores do processo de experimentação, além de influenciar na legitimidade conferida ao uso dessas bebidas ${ }^{(20)}$.

\section{CONSIDERAÇÕES FINAIS}

Os adolescentes participantes percebem a adolescência como um período conflituoso e difícil, onde a própria comunicação com outros sofre in- 
fluência das modificações biopsicossociais características deste período. A possibilidade de vivenciar novas experiências também foi apontada pelos jovens, independente do gênero, e foi possível compreender que experimentar o novo faz parte do processo de adaptação da transição entre a infância e a idade adulta.

Por meio da troca de conhecimentos entre adolescentes seus pares e pesquisadores, foi possível resignificar conhecimentos, o que pode vir a facilitar a aquisição de novas habilidades e atitudes para os participantes, frente a situações futuras que envolvam o uso de álcool. A ssim haverá o fortal ecimento da autonomia e empoderamento dos adolescentes frente às suas vidas.

E $m$ relação à percepção dos adolescentes acerca da associação entre o consumo de álcool e comportamento sexual, apontaram situações que evidenciam os riscos dessa díade, sendo o gênero masculino predominante em seus relatos. Salientam que beber facilita as relações interpessoais, nomeadamente com o par sexual, ficam mais descontraídos e sentem-se mais corajosos, o que pode facilitar o abuso desta substância provocando situações de risco e vulnerabilidade que influenciam negativamente na qualidade de vida.

As falas apontam que a mídia exerce influência importante, e é condutora ao consumo, influenciando o comportamento dos jovens. N este contexto, ela exerce papel preponderante por ser mediadora de informações, que muitas vezes pode ser inconsistentes e persuasivas a população juvenil, a qual muitas vezes despreparada, absorve as informações sem refletir sobre a real mensagem e conteúdo.

A família neste quesito deve ser reconheci$\mathrm{da}$, valorizada e fortalecida com vistas à influenciar positivamente a vida de seus membros adolescentes, pois é no seio familiar que o jovem recebe os primeiros ensinamentos para o viver saudável. É preciso juntar esforços, entre profissionais de saúde, educação, políticas de saúde, gestores e sociedade civil para o empoderamento da família, escola e comunidade para rompermos com o uso abusivo de álcool e suas consequências na vida de adolescentes no Brasil.

Ressaltamos que os achados aqui descritos não podem ser generalizados para toda a população adolescente, já que este estudo empregou um processo de amostragem por conveniência, ou seja, eles se referem ao grupo específico de jovens que participaram da pesquisa. N essa perspectiva os resultados contribuem para o entendimento da questão do comportamento sexual de risco associado ao consumo de bebidas alcoólicas por adolescentes, servindo, também, como ferramenta de construção de estratégias educativas em trabaIhos posteriores que visem à promoção da saúde desse grupo.

\section{REFERÊ NCIAS}

$1 \mathrm{M}$ inistério da Justiça (BR). Estatuto da Criança e do A dolescente: 12 anos. Brasília (DF ); 2002.

2 E spinosa M A, J ourdan G A M , L andmann SC. Padrão de comportamento relacionado às práticas sexuais e ao uso de drogas de adolescentes do sexo feminino residentes em V itória, Espírito Santo, Brasil, 2002. Cad Saúde Pública [ Internet] . 2005 [ citado 2009 jan 13] ; 21(1):207-16. Disponível em: http:/ / www.scielo.br/ $\mathrm{pdf} / \mathrm{csp} / \mathrm{v} 21 \mathrm{n} 1 / 23 . \mathrm{pdf}$.

3 M inistério do Planejamento, Orçamento e G estão (BR), Instituto Brasileiro de G eografia e E statística. Pesquisa nacional por amostra de domicílios: síntese de indicadores 2008.

4 Camargo BV, Bertoldo RB. Comparação da vulnerabilidade de estudantes da escola pública e particular em relação ao H IV. E stud P sicol [ I nternet] . 2006 [ citado 2010 mar 16];23(4). D isponível em: http:/ / pepsic.bvs-psi.org.br/ pdf/ epc/ v23n4/ v23n4a05.pdf.

5 Reboussin BA, Song EY, Shrestha A, Lohman KK, W olfson M. A latent class analysis of underage problem drinking: evidence from a community sample of 16-20 year olds. Drug Alcohol Depend. 2006;83(3): 199-209.

$6 \mathrm{G}$ alduroz JCF, N oto AR, Fonseca A M , Carlini EA.V levantamento nacional sobre o consumo de drogas psicotrópicas entre estudantes do ensino fundamental e médio da rede pública de ensino nas 27 capitais brasileiras: 2004. São Paulo: Centro Brasileiro de Informações sobre Drogas Psicotrópicas/ U N IFE SP; 2005.

7 Vilelas Janeiro J M S. Educar sexualmente os adolescentes: uma finalidade da família e da escola? Rev G aúcha E nferm. 2008;29(3):382-90.

8 Polit D F, Beck CT, H ungler BT. F undamentos da pesquisa em enfermagem: método, avaliação e utilização. Porto Alegre: A rtmed; 2004. 
9 M organ DL. Focus group as qualitative research. N ewbury Park: Sage; 1988.

10 Afonso L. Oficinas em dinâmica de grupo: um método de intervenção psicossocial. Belo Horizonte: Campo Social; 2000.

11 M inistério da Saúde (BR), Conselho Nacional de Saúde. Resolução 196, de 10 de outubro de 1996: diretrizes e normas regulamentadoras de pesquisa envolvendo seres humanos. Brasília (DF); 1996.

12 Gubert $F A$, Santos $A C L$, A ragão $K A$, Pereira $D C R$, Vieira NFC, Pinheiro PNC. Tecnologias educativas no contexto escolar: estratégia de educação em saúde em escola pública de F or tal eza-CE. Rev E letrônica Enferm [ I nternet] . 2009 [ citado 2010 mar 16] ; 11(1):165-72. Disponível em: http:// www.fen.ufg. br/ revista/ v11/ n1/ v11n1a21.htm.

13 Folle E, G eib LT C. Representações sociais das primíparas adolescentes sobre o cuidado mater no ao recém-nascido. Rev L atino-Am Enfermagem [ I nternet] . 2004 [ citado 2010 mar 14];12(2):183-90. Disponível em: http:// www.scielo.br/pdf/ rlae/ v12n2/ v12n2a06.pdf.

14 Brêtas JRS, et al. Os rituais de passagem segundo adolescentes. Acta Paul E nferm [ Internet] . 2008 [ citado 2009 jul 14];21(3):404-11. Disponível em: http:/ / www.scielo.br/ pdf/ ape/ v21n3/ 04.pdf.
$15 \mathrm{~N}$ úcleo de A poio e Prevenção a Aids (SP). Dados estatísticos do estudo na FEBEM Feminina e M asculina. São Paulo; 1992.

16 Dalen W E, van Kuunders M M AP. Alcohol marketing and young people: an analysis of the current debate on regulation. Nordic Stud Alcohol Drugs. 2006;23(6):415-26.

17 Pinsky I, Jundi S. 0 impacto da publicidade de bebidas al coólicas sobre o consumo entre jovens: revisão da literatura internacional. Rev Bras Psiquiatr [ I nter net] . 2008 [ citado 2009 jul 14] ;30(4):362-74. Disponível em: http:/ / www.abead.com.br/artigos/ arquivos/Artigo_0_impacto_publicidade_bebidas alcoolicas_sobre____jovens.pdf.

18 H astings G, Anderson S, Cooke E, G ordon R. Alcohol marketing and young people's drinking: a review of the research. J Public Health Policy. 2005;26(3): 292-5.

19 M inistério da Saúde (BR). Diretrizes e recomendações para o cuidado integral de doenças crônicas não-transmissíveis: promoção da saúde, vigilância, prevenção e assistência. Brasília (DF); 2008.

20 Abramovay M. Drogas nas escolas: versão resumida. Brasília (DF): U NESCO/Rede Pitágoras; 2005.

Recebido em: 14/ 04/ 2010

A provado em: 24/ 08/ 2010

\author{
Endereço da autora / Dirección del autor / \\ Author's address: \\ Fabiane do A maral Gubert \\ Rua A lexandre Baraúna, 1115, Rodolfo T eófilo \\ 60430-160, Fortaleza, Ceará \\ E - mail: fabianegubert@ hotmail.com
}

